\title{
Analysis and application of ATP7B gene mutations in 35 patients with hepatolenticular degeneration
}

\author{
Y.N. Zong and X.D. Kong \\ Genetics and Prenatal Diagnostic Center, Zhengzhou, China \\ Corresponding author: X.D. Kong \\ E-mail: kongxd@zzu.edu.com \\ Genet. Mol. Res. 14 (4): 18764-18770 (2015) \\ Received August 11, 2015 \\ Accepted October 7, 2015 \\ Published December 28, 2015 \\ DOI http://dx.doi.org/10.4238/2015.December.28.25
}

ABSTRACT. We investigated the genetic mutations involved in Wilson's disease to improve prenatal genetic diagnosis and presymptomatic diagnosis. The polymerase chain reaction ( $P C R$ ) was used to amplify the exons and exon-intron boundaries of the ATP7B gene in 35 Wilson's disease pedigrees. The PCR products were further analyzed by Sanger sequencing. Prenatal genetic diagnoses were performed by chorionic villus sampling after the genotypes of parents of the probands were identified. The overall mutation detection frequency was $92.9 \%$. A total of 24 distinct mutations were detected, seven of which are novel: A1291T (c.3871G>A), c.2593_2594insGTCA, c.2790_2792delCAT, c.3661_3663delGGG, c.3700delG, c.4094_4097delCTGT, and IVS6+1G>A. Three mutations, R778L (c.2333G>T) (45.7\%), A874V (c.2621C>T) (7.1\%), and P992L (c.2975C>T) (7.1\%) are relatively frequent. Two presymptomatic patients were detected through familial screening, and they began taking medicine after diagnosis. Of the subjects with Wilson's disease pedigrees who had received a prenatal genetic diagnosis, three fetuses were normal and one was a carrier. Twenty-four distinct mutations were identified, and our knowledge of the population genetics of Wilson's disease in China has therefore improved. For pedigrees with the Wilson's disease, genetic 
counseling, prenatal diagnosis, and presymptomatic diagnosis by Sanger sequencing and haplotype analysis are feasible.

Key words: Wilson's disease; ATP7B gene; Mutation analysis; Prenatal diagnosis; Presymptomatic diagnosis

\section{INTRODUCTION}

Hepatolenticular degeneration, also known as Wilson's disease, is an autosomal recessive disorder that was first described in 1912 (Kinnier Wilson, 1912). The incidence rate in the general population is $1 / 30,000-1 / 100,000$, and the probability of recessive carriers is $1 / 90$ (Ala et al., 2007). The gene that causes hepatolenticular degeneration is ATP7B. It has the chromosomal locus $13 q 14.3$, is about $80 \mathrm{~kb}$, contains 21 exons, encodes ATP7B proteins, and is involved in the process of copper transmembrane transport in the body. ATP7B gene mutations lead to abnormal $\mathrm{Cu}^{2+}$ excretion and excess $\mathrm{Cu}^{2+}$ deposition in organs such as the liver, brain, and cornea, resulting in the corresponding pathologies. There is no ideal therapeutic remedy for the disease (Ala et al., 2007). Children may progressively develop cirrhosis and liver cancer, which can be ultimately fatal, and the neurological symptoms seriously affect the patient's quality of life. Currently, presymptomatic and prenatal diagnoses are carried out by gene detection, which is still the most effective way of preventing hepatolenticular degeneration (Huang et al., 2008).

To date, ATP7B gene mutations have been found in more than 500 species (Li et al., 2011). Mutation characteristics have been reported in an increasing number of races, as well as in China (Du et al., 2008; Huang et al., 2008; Li et al., 2011; Wang et al., 2011), but most of the study subjects were patients with hepatolenticular degeneration from the southern region. From 2009 to December 2014, 35 probands with hepatolenticular degeneration pedigrees carrying the 21-exon ATP7B gene were identified by Sanger sequencing in our center. The mutation characteristics of the probands were analyzed; seven pedigrees were detected by presymptomatic diagnosis and four pedigrees by prenatal diagnosis. We report our finding here.

\section{MATERIAL AND METHODS}

\section{Subjects}

Thirty-five probands with clinically diagnosed hepatolenticular degeneration from Henan, Shandong, Shaanxi, and other northern areas participated in this study. All were Han nationality, and no subjects were related by consanguineous marriage. All cases were diagnosed in the neurology, digestive system, or pediatrics departments, according to the clinical guideline for hepatolenticular degeneration proposed by Roberts et al. (2003). The subjects also include parents of probands from 31 pedigrees, and 10 proband compatriots from 7 pedigrees. This study was approved by the medical Ethics Council of the First Affiliated Hospital of Zhengzhou University. All participants voluntarily joined this genetic diagnostic study and gave their informed consent.

\section{Methods}

We extracted DNA from the peripheral blood of the probands and pedigree members, and obtained fetal villi from pregnant woman in the 11-13th week of pregnancy by abdominal puncture 
the guidance of under the guidance of ultrasound. We used TIANGEN kit to extract genomic DNA (gDNA). We determined DNA concentration and purity using a NanoVue ${ }^{\mathrm{TM}}$ Plus protein and nucleic acid quantitative spectrophotometer (GE Healthcare).

\section{Polymerase chain reaction (PCR) amplification}

We amplified 21 exons of the ATP7B gene using the primer sequence, reported in the literature (Thomas et al., 1994), which was synthesized by Shanghai Sangon Biological Engineering Technology \& Services Co., Ltd. A conventional PCR system and conventional reaction conditions were used. The detected PCR products were recorded using the QIAxcel Advanced automated capillary electrophoresis apparatus (QIAGEN).

\section{Sanger sequencing of purified PCR products}

We used an ABI PRISM BigDye 3.1 sequencing kit (Applied Biosystems, USA) and an ABI PRISM 3130xl genetic analyzer (Applied Biosystems) to sequence the PCR products, and the results were compared with the normal sequence by the Chromas software, searching for point mutations. We initially identified novel mutations by exploring the literature and the Human Gene Mutation Database (HGMD) (http://www.hgmd.cf.ac.uk/ac/index.php).

\section{Prenatal diagnosis and exclusion of polluted fetal tissue}

We used Sanger sequencing to analyze fetal $A T P 7 B$ gene mutations. To exclude fetal tissue contaminated by the maternal tissue, we used a PowerPlex 16 HS kit (Promega). The PCR amplification instructions were followed and the PCR product was investigated using a gene analyzer; the GeneMapper4.0 software (Applied Biosystems) was used for analysis.

\section{Linkage analysis}

We selected five short tandem repeat (STR) polymorphisms for linkage analysis. We used the primer sequences and PCR conditions reported in the literature (Thomas et al., 1994), and the PCR products were detected and analyzed.

\section{Follow-up}

After the babies were born, we took umbilical cord blood specimens for prenatal diagnosis, and carried out follow-up on each baby's condition after 1 year.

\section{RESULTS}

\section{ATP7B gene Sanger sequencing}

Thirty-five probands (number of alleles, $N=70$ ) were detected in 65 pathogenic alleles (Table 1), with a mutation detection rate of $92.9 \%$. In 31 cases, two allelic mutations were detected (homozygous mutation: nine cases; compound heterozygous mutation: 22 cases). In three cases, 
one mutation was detected, and no mutations were detected in one case. In this study, 24 mutations were detected, including 13 missense mutations, three nonsense mutations, four small deletions, one small insertion mutation, and three splice site mutations. R778L (45.7\%), A874V (7.1\%), and P992L (7.1\%) were the most common mutations, and 19 mutations were detected only once. In this study, there were seven previously unreported mutations: A1291T, c.4094_4097delCTGT, c.2593_2594insGTCA, c.2790_2792delCAT, c.3700delG, c.3661_3663delGGG, and IVS6+1G>A (Figure 1). None of these mutations was detected in the 100 normal controls.

We also detected 13 single nucleotide polymorphisms: -75C>A, I390V, S406A, V456L,

Table 1. ATP7B gene mutations and allele frequencies in the 35 probands with Wilson's disease.

\begin{tabular}{|c|c|c|c|c|c|}
\hline Nucleotide change & Amino acid change & Mutation type & Exon & Number of mutations & Allele frequency $(N=70)$ \\
\hline c. $1475 \mathrm{~T}>\mathrm{C}$ & L492S & Missense mutation & E3 & 1 & $1.4 \%$ \\
\hline c. $2333 \mathrm{G}>\mathrm{T}$ & $\mathrm{R} 778 \mathrm{~L}$ & Missense mutation & E8 & 32 & $45.7 \%$ \\
\hline c. $2621 \mathrm{C}>\mathrm{T}$ & A874V & Missense mutation & E11 & 5 & $7.1 \%$ \\
\hline c. $2620 \mathrm{G}>\mathrm{C}$ & A874P & Missense mutation & E11 & 1 & $1.4 \%$ \\
\hline c. $2827 \mathrm{G}>\mathrm{A}$ & G943S & Missense mutation & E12 & 1 & $1.4 \%$ \\
\hline c. $2924 \mathrm{C}>\mathrm{A}$ & S975Y & Missense mutation & E13 & 1 & $1.4 \%$ \\
\hline c. $2930 \mathrm{C}>\mathrm{T}$ & T977M & Missense mutation & E13 & 1 & $1.4 \%$ \\
\hline c. $2975 \mathrm{C}>\mathrm{T}$ & P992L & Missense mutation & E13 & 5 & $7.1 \%$ \\
\hline c. $3188 \mathrm{C}>\mathrm{T}$ & A1063V & Missense mutation & E14 & 1 & $1.4 \%$ \\
\hline c. $3316 \mathrm{G}>\mathrm{A}$ & V1106I & Missense mutation & E15 & 1 & $1.4 \%$ \\
\hline c. $3517 \mathrm{G}>\mathrm{A}$ & G1173K & Missense mutation & E16 & 2 & $2.9 \%$ \\
\hline c. $3646 \mathrm{G}>\mathrm{A}$ & V1216M & Missense mutation & $\mathrm{E} 17$ & 1 & $1.4 \%$ \\
\hline c. $3871 \mathrm{G}>\mathrm{A}^{*}$ & A1291T & Missense mutation & E18 & 1 & $1.4 \%$ \\
\hline c. $3955 \mathrm{C}>\mathrm{T}$ & R1319X & Nonsense mutation & E19 & 1 & $1.4 \%$ \\
\hline c. $2038 \mathrm{C}>\mathrm{T}$ & Q680X & Nonsense mutation & E7 & 1 & $1.4 \%$ \\
\hline c. $4114 C>T$ & Q1372X & Nonsense mutation & E20 & 1 & $1.4 \%$ \\
\hline c.2593_2594insGTCA* & & Insertion mutation & E11 & 1 & $1.4 \%$ \\
\hline C.2790_2792delCAT* & & Deletion mutation & E12 & 1 & $1.4 \%$ \\
\hline C. $3700 \mathrm{delG}^{*}$ & & Deletion/splice site & E18 & 2 & $2.9 \%$ \\
\hline C.3661_3663delGGG* & & Deletion mutation & $\mathrm{E} 17$ & 1 & $1.4 \%$ \\
\hline c.4094_4097delCTGT* & & Deletion mutation & E20 & 1 & $1.4 \%$ \\
\hline IVS4-1G $>C$ & & Splice site mutation & & 1 & $1.4 \%$ \\
\hline IVS4-5T>G & & Splice site mutation & & 1 & $1.4 \%$ \\
\hline IVS $6+1 G>A^{*}$ & & Splice site mutation & & 1 & $1.4 \%$ \\
\hline A combined & & & & 65 & $100.0 \%$ \\
\hline
\end{tabular}

${ }^{*}$ Represent newly discovered mutations.
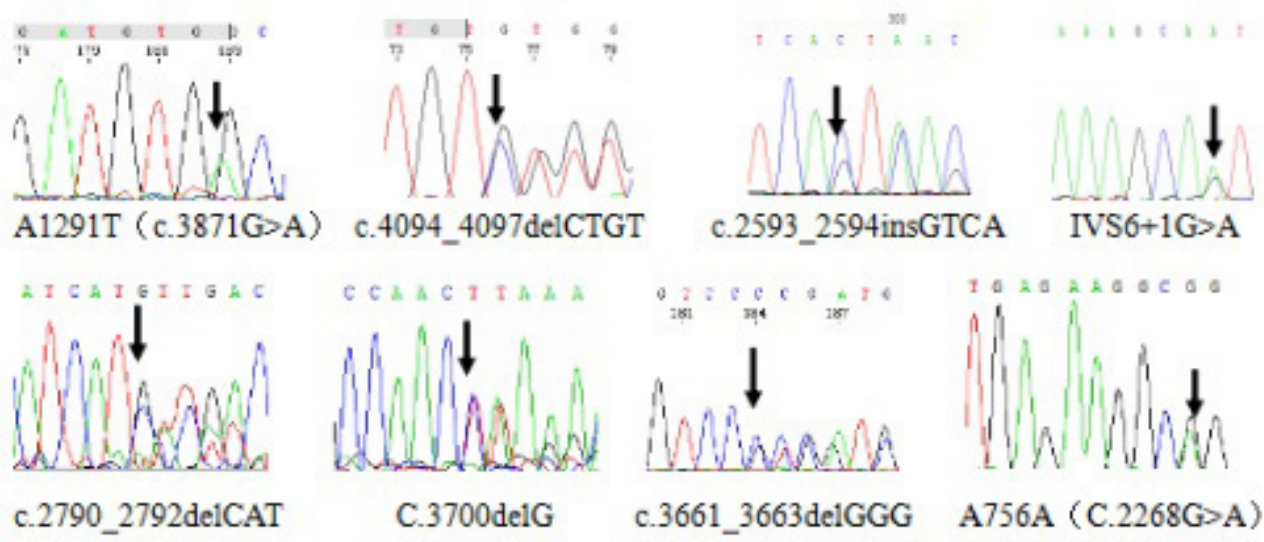

A756A (C.2268G >A)

Figure 1. Sequencing diagram showing seven newly discovered mutations and a new single nucleotide polymorphism A756A. The arrows represent the mutation positions. 
A756Am, L770L, K832R, R952K, V1140A, IVS9-25G>A, IVS15-4A>T, IVS18+6C>T, and IVS19+50G >C. A756A had not been reported before (Figure 1).

\section{Presymptomatic diagnosis}

After presymptomatic examination, we found the pedigree 13 proband's younger brother (genotype R778L/ c.2790_2792delCAT), and the pedigree 22 proband's younger sister (genotype L492S/A874V) were consistent with the proband genotype of the presymptomatic states. Serum ceruloplasmin, serum copper, and urinary copper levels were all abnormal, but improved following drug treatment after detection.

\section{Prenatal diagnosis}

The probands of pedigrees 4,5 , and 6 all had R778L homozygous mutations, and their parents were heterozygous carriers. The proband of pedigree 28 had an R778L/P992L compound heterozygous mutation; his father carried the R778L mutation and his mother carried the P992L mutation. The fetuses of pedigrees 4,5 , and 28 had the homozygous wild-type genotype. Six fetuses of pedigree 6 were R778L heterozygous carriers. Linkage analysis showed that the fetuses of pedigrees 4,5 , and 28 did not carry any risk of chromosomal disorders from their parents. Linkage analysis of pedigree 6 showed that the fetuses acquired risk of chromosomal disorders from their parents, which was consistent with the sequencing results. After genetic counseling, four couples chose to continue the pregnancy; blood taken from the umbilical cords after the babies were born gave results that were consistent with the prenatal diagnosis.

\section{DISCUSSION}

Missense and nonsense mutations were the most common ATP7B gene mutations, followed by small fragment insertion deletions and splice site mutations. Mutations were mainly found in a few hot spots, and there was a large number of rare mutations. Different regions and ethnic groups have different patterns of mutation. In Europe, the most common mutation is $\mathrm{H} 1069 \mathrm{Q}$ (Ferenci, 2006), and the most common mutation in Asia is R778L (Thomas et al., 1994). Jiao et al. (2009) reported that the mutation rate of $\mathrm{R} 778 \mathrm{~L}$ was $40.78 \%$ in 146 cases of hepatolenticular degeneration. In this study, the mutation rate of $\mathrm{R} 778 \mathrm{~L}$ was $45.7 \%$. The ATP7B mutation is mainly expressed in the liver, and encodes an approximately $160-k D a$ protein located in the Golgi membranes (Ala et al., 2007). The protein includes the following five functional areas: a copper ion-binding region; an adenosine triphosphate (ATP)-binding domain; a transduction domain; a transmembrane cation channel/phosphorylation area; and a transmembrane region. In this study, seven new mutations were detected. A1291T, a missense mutation located in the ATP-binding domain of the ATP7B protein, represented the conversion of the neutral amino acid alanine to the aromatic amino acid tryptophan. Software was used to predict the effect of a deleterious mutation (PolyPhen2 software gave a predictive value of 0.922; PROVEAN software gave a predictive value of -3.625). Mutations c.2790_2792delCAT and c.2593_2594insGTCA, which result in frameshift mutations, produce proteins that are located in the 5th transmembrane domain, are infered to result in frameshift mutations. The c.3661_3663delGGG mutation, which is a codon mutation resulting in the deletion of the 1221 th glycine, produces a protein located in the ATP-binding domain. The 
c.4094_4097delCTGT mutation, which is a frameshift mutation, produces a protein located in the 8th transmembrane domain, resulting in ATP7B protein truncation; and the c.3700delG mutation, located in the first basic group of the 18th exon, and IVS6+1G>A, located in the shear zone, probably affect mRNA splicing. It is speculated that the mutations mentioned above are pathogenic in nature, but further studies are required on their expression in vitro to determine the specific pathogenesis.

Hepatolenticular degeneration is a treatable hereditary disease, but most patients start treatment after the onset of illness, and the drug used to treat the disorder does not completely restore liver function following the removal of copper - it may even aggravate neurological symptoms. Some patients eventually need a liver transplant (Ala et al., 2007), so the best prevention is to avoid conception or perform presymptomatic diagnosis.

The European Association for the Study of the Liver (EASL) guidelines (2012) recommend that first-degree relatives of patients with hepatolenticular degeneration (compatriots or children) undergo genetic screening. However, there are few reports in the literature of illness in the parents of probands, although the probands' parents also have a $0.5 \%$ risk of developing the disease. In view of the fact that patients range in age from 2 to 70 years, there are some late-onset patients, and therefore it is important to screen for presymptomatic states in pedigrees as part of early diagnosis and treatment. In this study, two cases of presymptomatic states were diagnosed with screening, and early treatment can significantly improve the symptoms.

Couples with a childbearing or family history of Wilson's disease usually have a strong desire for prenatal diagnosis when they have an additional child. In this study, we extracted fetal villi from women in the 11-13th week of pregnancy and examined the samples using Sanger sequencing, which could be used for prenatal diagnosis in one week. However, prenatal genetic diagnosis needs to define the genotypes of the proband and parent, and exclude maternal blood contamination. In addition, Sanger sequencing and linkage analysis can proceed simultaneously, so that the results of the genetic diagnosis can be verified with each other, improving diagnostic accuracy.

\section{Conflicts of interest}

The authors declare no conflict of interest.

\section{ACKNOWLEDGMENTS}

Research the Health Department of Henan Province Scientific and Technological Projects (\#201303015).

\section{REFERENCES}

Ala A, Walker AP, Ashkan K, Dooley JS, et al. (2007). Wilson's disease. Lancet 369: 397-408.

Du J, Gao B, Li L, Li W, et al. (2008). Mutation screening and prenatal diagnosis of Wilson's disease by denature high performance liquid chromatography. Zhonghua Yi Xue Yi Chuan Xue Za Zhi. 25: 527-530.

European Association for the Study of the Liver (EASL) (2012). EASL Clinical Practice Guidelines: Wilson's disease. J. Hepatol. 56: 671-685.

Ferenci P (2006). Regional distribution of mutations of the ATP7B gene in patients with Wilson disease: impact on genetic testing. Hum. Genet. 120: 151-159.

Huang LS, Liu XQ, Jiao XT and Zhang YF (2008). Prenatal and pre-symptomatic diagnosis of Wilson disease. J. Clin. Pediatr. 26: 955-958. 
Jiao XT, Liu XQ, Zhang YF, Wu J, et al. (2009). Study on mutation in exon 8 of ATP7B gene in Chinese patients with Wilson disease. J. Appl. Clin. Pediatr. 24: 575-579.

Kinnier Wilson SA (1912). Progressive lenticular degeneration: a familial nervous disease associated with cirrhosis of the liver. Brain 34: 295-507.

Li XH, Lu Y, Ling Y, Fu QC, et al. (2011). Clinical and molecular characterization of Wilson's disease in China: identification of 14 novel mutations. BMC Med. Genet. 12: 6.

Roberts EA, Schilsky ML and Division of Gastroenterology and Nutrition, Hospital for Sick Children, Toronto, Ontario, Canada. (2003). A practice guideline on Wilson disease. Hepatology 37: 1475-1492.

Thomas GR, Bull PC, Roberts EA, Walshe JM, et al. (1994). Haplotype studies in Wilson disease. Am. J. Hum. Genet. 54: 71-78.

Wang LH, Huang YQ, Shang X, Su QX, et al. (2011). Mutation analysis of 73 southern Chinese Wilson's disease patients: identification of 10 novel mutations and its clinical correlation. J. Hum. Genet. 56: 660-665. 